\title{
Turning Back the Clock: The Effect of the COVID-19 Response in Sub-Saharan Africa and Other Low- Income Countries
}

\author{
Christopher A Rice and Sanghvi Reema* \\ Department of Anesthesiology, UC San Diego School of Medicine, USA
}

*Corresponding author: Sanghvi Reema, Department of Anesthesiology, UC San Diego School of Medicine, USA.

Received Date: May 04, 2021

Published Date: June 15, 2021

\section{Introduction}

The COVID-19 pandemic has affected everyone across the world, either through the disease itself or with our response to the disease as healthcare professionals, for the better part of a year. In the matter of a few months, it managed to spread from its little corner of the world to a true pandemic. In response to this global pandemic many affluent nations have instituted lockdown procedures to protect their population, a practice that has been adopted by many low to low- middle income countries (LMICs) as well.

Unfortunately, this pandemic does not take place in a vacuum, and individuals can have more than one condition with resultant needs outside their homes. This becomes readily apparent when considering someone receiving medical treatment for a disease such as HIV or TB, who may struggle keeping their treatment appointments. It also can restrict patients who rely on public transportation to reach hospitals or clinics, for example, pregnant women attempting to see their obstetrician or when going into labor. A lockdown of this magnitude and duration, while bothersome in wealthy countries, can be catastrophic in LMICs. In addition to access to medical care, this lockdown can also have an effect on a family's financial stability. Many work in the informal economy even a day without work could lead to an inability to place food on the table [1].

Many individuals in these countries live in overcrowded spaces, leading to local outbreaks within a community. Enforcement of the lockdown has also seen an increase in violence towards those violating the order. In this review we will examine the literature, with a few firsthand accounts as well, of how the pandemic is currently being handled. We will examine the multitude of effects these policies are having on the local populace, particularly in Sub-Saharan Africa, the socioeconomic impact it is having, and hopefully elicit agreed upon recommendations for how national health agencies could create an individualized approach to their own COVID-19 response. We will also highlight areas that would benefit from additional research in the coming months to years.

\section{Background}

As of August 2020, there had been over 19 million confirmed cases of COVID-19 and 716 thousand deaths worldwide. By the end of 2020 this jumped to 81.9 million cases and 1.8 million deaths [2]. Through 2020 a little less than half of these cases occurred in the Americas, the United States leading with 19.6 million cases and 341 thousand deaths [3]. By contrast, all of Africa has 1.9 million cases with 42 thousand deaths. This all translates to 86.6 deaths per 100,000 in the Americas and only 4.2 per 100,000 in Africa. It is also important to point out that, for example, in the country of Malawi the average age is 17 with only $6.6 \%$ of the population over the age of 60 [4]. In comparison, in the United States the median age is 38.5 with $16.9 \%$ greater than the age of 65 , in the United Kingdom the median age is 40.6 with $18.5 \%$ greater than the age of 65 , and in Japan the median age is 48.6 with $29.2 \%$ over the age of 65 [5]. 
This should be an important consideration seeing that advanced age is a significant risk factor for morbidity and mortality due to this disease. The response to this pandemic is constantly changing based on recommendations from the various international health organizations. As areas are hit harder by the disease, restrictions there may increase and vice versa. Nations have adopted their own policies in how to combat this pandemic from quarantining the sick to full on lockdown. It has been speculated and even shown that minimizing interactions within a population, along with better hygiene practices, can slow the spread of disease, the so-called flattening of the curve. This is of course a good thing for health care systems, and the more well-developed nations have benefited from this practice. The problem lies when considering how this could affect the developing world [6].

Many LMICs have also adopted these lockdown policies while their health care systems are significantly lacking compared to wealthier countries. There is evidence that non-COVID-19 deaths (such as cancer deaths, measles, women dying in labor) are increasing due to disruption in health services [6]. It has been postulated that lives lost to lockdown could potentially exceed those saved from COVID-19. In Africa patients rely on their national health services or non- government organizations (NGOs) for HIV and TB treatment programs which can face interruptions in access to treatment. Other potentially fatal unintended consequences of lockdown include hunger, food insecurity, and violence [4].

Some sources question the feasibility of the high-income country model in LMICs, stating that this blueprint could negatively affect the economy and food systems, access to education and routine clinical services, the burden of vaccine-preventable diseases, and could even be counterproductive with regards to COVID-19 spread due to lockdowns causing out-migration from cities [7]. In India, many informal workers live in outlying villages while working in large city centers [8]. When the lockdown was put into effect, public transportation shut down almost overnight and these individuals, deprived of their livelihood, were left with no other options but to walk for days, sometimes covering hundreds of miles, risking death just to escape the city and return home to their families.

\section{COVID-19 Compared to other Pandemics}

While not the focus of this review, it is still important to look back briefly at some other pandemics we have encountered. COVID-19 is unfortunately just the latest pandemic faced by humanity, with notable predecessors such as the H1N1 swine flu of 2009-2010, the related coronavirus SARS pandemic of 2002, the 1918 Spanish flu, and of course The Black Death of the 14th century [9]. The swine flu, while viral like SARS-CoV-2, has an interesting difference in that some of the older population had some immunity, likely due to infection from a similar strain decades before. This made $62-85 \%$ of swine flu fatalities in patients less than 65 [10]. COVID-19 is also more highly transmittable with a Ro of 1.6-2.6 [11] while the swine flu was 1.4- 1.6 [10]. The 2002 SARS pandemic was also caused by a coronavirus, SARS-CoV, also originating in China. These have a similar transmission and patient presentation with most fatalities in the elderly over 65, however COVID-19 does appear more infectious with more fatalities to date.

SARS was eventually eradicated through surveillance, isolation, and quarantine [12]. It is apparent with these few examples that these various pandemics share a lot of similarities and some notable differences. For example, The Black Death was caused by a bacterium while the others on this list are caused by viruses. However, one similarity shared between all these pandemics is the disproportional impact they have on vulnerable populations such as the old, the sick, and the poor. A lesson learned from these prior pandemics is the effectiveness of surveillance and quarantine. However, as the rest of this review will illustrate, it is important not to just quarantine an entire community. We must first consider the full effect it will have on the populace and ensure steps have been taken to address the aptly named lockdown effect.

\section{Coexisting Conditions Requiring Regular Treatment}

As mentioned earlier, COVID-19 does not exist in a vacuum. Individuals can and do have more than one condition affecting their overall health. In sub-Saharan Africa tuberculosis, malaria, and HIV/ AIDS had been a large focus of NGOs and national healthcare systems, and prior to the emergence of SARS-CoV-2 they had been working diligently to combat these diseases. They were accomplishing this by working to lower active and new infections now and in the coming years. These plans are now directly being jeopardized by the emergence of this new pandemic. Various reports are showing disruption of healthcare services, diversion of the workforce, and travel/supply chain disruption, all due to the COVID-19 response and various lockdowns [13]. The WHO notes that during the recent Ebola outbreak in west Africa there was an increase in other disease morbidity and mortality with the sudden increase in demand for health services [14]. The importance of ensuring continued access to care for these diseases, especially during the currently year-long COVID-19 pandemic, cannot be understated. It is also pertinent to note that while TB, malaria, and HIV/AIDS are well known in this region, other infection prevention campaigns are also affected. For example, according to a special report in Nature [15], measles rates have been declining for the past 40 years but due to concerns for COVID-19 over 20 countries have suspended vaccination campaigns and measles rates are projected to rise. This is especially concerning in a country like the Democratic Republic of the Congo as it has the greatest single nation outbreak of measles in decades, with an estimated 6,500 child deaths from 2019-20, and as of March 2020 projections are continuing to rise [15]. One highimpact scenario predicts 84 immunization preventable deaths in children in Africa for everyone excess COVID- 19 death attributed to infection acquired during routine vaccine clinic visits, which are shut down in the interest of quarantining [16]. However, in this review we will focus on tuberculosis, malaria, and HIV/AIDS 
as examples of the effect COVID-19 is having on combating other diseases.

\section{Tuberculosis}

Tuberculosis (TB) is a well-known bacterial infection seen worldwide with well documented treatment and prevention strategies. While seen across the world, around 20 countries, especially in Africa, south Asia, and south-east Asia, are collectively known as high-burden countries that make up 54\% of the global TB burden [17]. The Stop TB Partnership performed a rapid assessment and modeling analysis of the impact COVID-19 and the associated lockdown are expected to have on TB in the coming months to years. Looking across 16 high-burden countries they noted at least $40 \%$ of TB facilities being utilized for COVID-19 responses [17]. In India they have noticed a decrease by $80 \%$ of daily TB notifications during the lockdown contributed to people avoiding or being unable to reach medical care, laboratory delays, and stoppage of case finding actives [17]. They note not only lack of access to testing but also lack of medicines with no time for hospitals to prepare in advance for curfews and lack of patient transportation [17]. The modeling report was focused on three countries in particular; India, Kenya, and Ukraine; with their results extrapolated to a global level. When looking at the estimated impact over the next 5 years the study showed upwards of $10.7 \%$ increase in cases and a $16 \%$ increase of deaths between 2020-2025 when considering a 3-month lockdown with 10-month recovery of services [18]. They also estimated for every month of lockdown they expect over 600,000 more cases and over 125,000 more deaths; with every month of recovery, they expect over 400,000 more cases and over 80,000 more deaths [18]. In summary they have determined a setback of 5-8 years in the fight against TB due to the increase in incidence and deaths due to the COVID-19 pandemic [18]. This illustrates the importance of ensuring continued access to care for patients with TB living and receiving treatment within locked down communities. TB requires months of antibiotic treatments, access and transportation to regular medical care, and timely recognition of new cases; all things directly impacted by a lockdown. While no one expected nor was prepared for such a lockdown steps need to be taken to ensure continued access to TB treatment throughout this pandemic.

\section{Malaria}

The case incidence rate of malaria has decreased by $30 \%$ from 2000 to 2018 while the case mortality has decreased by $60 \%$ over the same period, the majority of which has occurred in subSaharan Africa, the area of the world that accounts for $90 \%$ of global malaria cases [14]. The WHO had previously developed a modeling framework detailing the normalized malaria incidence per person year from 2016 to 2030, a model they used as the basis of their COVID-19 model. Prior to developing their model, they determined the primary disruptions in intervention secondary to the COVID-19 response to be distribution of insecticide treated nets, indoor residual spraying, seasonal malaria chemoprevention, and access to malaria diagnosis and treatment. They then looked at 9 different possible scenarios with differences in reduction of net campaigns, distribution, and available effective treatment. Examples of some of these scenarios include scenario 1 in which they assumed no net campaigns and continuous net distribution decreased by $25 \%$. In scenario 4, only effective antimalarial treatment was assumed to be reduced by $25 \%$. Finally, in scenario 9 they assumed no net campaigns and a $75 \%$ reduction in net distribution and effective treatment. The remaining six scenarios fell along a spectrum similar to these three. While considering possible effect, in scenario 9 they concluded that every country in sub-Saharan Africa would see at least $20 \%$ increase in malaria deaths in 2020 compared to 2018 , with the highest being greater than $200 \%$ increase in malaria deaths specifically in Guinea Bissau and Uganda [14]. In addition, the WHO published recommendations for malaria intervention in the setting of COVID-19 with guidance for vector control, case management, chemoprevention, and other extraordinary interventions [19]. Malaria is a prime example of a disease that has seen great reductions in recent years and, prior to the emergence of COVID-19, continued improvement was expected. The WHO model looks at nine different scenarios that could play out in the coming months, all of which illustrate an increase in both malaria cases and mortality in the setting of COVID-19. Much like the other diseases analyzed in this paper, the importance of continued access to malaria care by healthcare systems needs to be maintained. Fortunately, in the case of malaria, the WHO has published comprehensive recommendations that can help guide healthcare systems in developing their COVID-19 response with regards to malaria, which can be found on the WHO website at https://www. who.int/publications/m/item/tailoring-malaria-interventions-inthe-covid-19-response.

\section{HIV/AIDS}

COVID-19 may be our current pandemic, but the HIV/AIDS pandemic was here long before COVID-19 and will continue to plague society after it is gone. There is not a corner of the world that has not experienced HIV/AIDS to some degree. It is the most wellknown pandemic prior to the one we currently find ourselves in, and it is also probably the best studied. Researchers have spent decades studying and developing therapies against HIV/AIDS while various national and international organizations have spent time and money combating this disease. As of 2018, there were 37.9 million people living with HIV, according to UNAIDS two-thirds of those live in sub- Saharan Africa [20]. The WHO and UNAIDS used 5 existing HIV models to determine the potential effect that disruptions of access to care due to the COVID-19 pandemic will have on prevention and treatment. The various models examined how disruptions in specific HIV related services would affect incidence and mortality over both and 1- and 5-year period. Some of the services they considered were condom availability, suspension of HIV testing, no new anti- retroviral therapy (ART) initiation, stoppage of viral 
load testing and adherence counseling services, ART interruption, and others [20]. Across the models they found a $1.87-2.80$-fold increase in HIV related deaths after only a six-month interruption of antiretroviral drug supply [20]. For example, in Kenya there were an estimated 25,000 HIV-related deaths in 2018. The five models examined saw an increase of 32,000 - 58,000 excess HIV-related deaths over 1 year as compared to 2018 data [20], with similar trends in all other African nations. The total sub- Saharan Africa excess HIV deaths over 1 year after a 6-month interruption ranged from 471,000-673,000 based on the model examined [20]. During the current COVID-19 pandemic it is important to prioritize where the time and resources should go concerning HIV/AIDS. Based on the results in this study it appears that the most important service to ensure reduced interruptions is ART. Maintaining as many HIV/ AIDS related services as possible, such as prevention and testing, are also important yet ART interruption would have the largest effect. Therefore, it is important for healthcare systems and NGOs in sub-Saharan Africa to ensure continued access to anti-retroviral medications during this COVID-19 pandemic.

\section{Effects on Pregnancy and Women's Health}

There is no question that while we all have the potential for exposure to SARS-CoV-2 there are certain populations that we consider at greater risk for infection and disease course. As previously mentioned, the poor, the sick, and the old all fall into that category. However, there is increasing evidence that some women should be considered at greater risk as well. While pregnancy is the most obvious contributor to this claim, things such as employment opportunities, domestic violence, and access to sexual and reproductive health (including pregnancy, family planning, and availability of contraception) all play a role. One report looking at 118 LMICs estimate a worse-case scenario of 1,157,000 additional child deaths and 56,700 additional maternal deaths over the first six months due to disruptions in access to routine healthcare and food security [21]. Per the UN policy brief it is known that women are $25 \%$ more likely to live in poverty globally compared to men [22] and are also $1 / 3$ rd more likely than men to work in a sector now closed due to lockdown. This includes non-food retail, restaurants and hotels, childcare, arts and leisure, and personal care [23]. There is also the concern of domestic violence. The UN believes that the number of women and girls subjected to sexual and/or physical violence is expected to increase in the setting of the lockdown. At the time of publishing in April 2020 there was a documented increase of domestic violence in France of 30\% and Argentina of 25\% since lockdown a month prior [22]. The Center for Global Development documented nine pathways based on published literature linking pandemics to increased violence against women and children including quarantines and social isolation, reduced health service availability and access to first responders, and inability of women to temporarily escape abusive partners to name a few [23].

With regards to a woman's sexual and reproductive health it is believed that COVID-19 will cause disruptions in access to care and availability of family planning and contraceptive services. Per UN News, in 114 LMICs there are approximately 450 million women using various forms of contraception, anticipating that six months of lockdown would lead to 47 million without access to contraception and 7 million unintended pregnancies [25]. There is evidence of this during the outbreak of the HIV pandemic, where women's access to reproductive health care and family planning services was limited [26]. Pregnancy also places women and their fetuses at high-risk. It is well established that physiological changes during pregnancy increases risk of infection, in general, and dominance of the $\mathrm{T}$ helper cell type 2 system during pregnancy decreases a woman's defense against viral infections such as SARS-CoV-2, as viral defense is primarily T helper cell type 1 dominant [27]. While complications during pregnancy seem to be less serious when compared to SARS and MERS there is still a $\%$ risk of miscarriage, $10 \%$ risk of intrauterine growth restriction, and 39\% increase of preterm birth seen with COVID-19, however, a mild fever is the most common symptom [27]. With regards to vertical transmission there is limited data. In a study looking at close to 50 neonates born to COVID-19 positive mothers, two tested positive while the others did not [27]. Most of these mothers became infected during the third trimester. While data is limited, one case report of a severe case of COVID-19 in a 41-year-old G3P2 at 33 weeks gestation is worth mentioning. The mother presented with 4 days of mild symptoms that then progressed to respiratory failure requiring mechanical ventilation on day 5 . She was nasal swab positive for SARS-CoV-2 and negative on serology.

The mother then underwent Cesarean delivery with no delayed cord clamping or skin-to-skin. The neonate was intubated and at 16 hours had a positive nasal swab for SARS-CoV-2, required mechanical ventilation for 12 hours, and then had mild symptoms requiring supplemental oxygen on day six of life [28]. Data is limited with regards to vertical transmission, so it is not entirely clear whether it is occurring or not. However, the vulnerability of both mother and fetus/neonate place both individuals in a highrisk group requiring increased precautions. Protecting women's access to healthcare services and certain other protections must be prioritized, without lapses, during this and future pandemics. Maintaining reporting pathways and protections to those facing abuse is imperative, along with financial protections to those facing poverty due to the lockdown. While not specific just to women's health, many countries' lockdowns affect and even shutdown public transportation, making it difficult if not impossible to get to a healthcare institution, even if the clinic is open. While data is limited, there are firsthand accounts and news reports out of countries like Uganda of women in labor dying while attempting to reach hospitals by foot due to lack of ambulances and public transport [7].

Pregnant women face the increased risk of infection, not just for themselves, but for their fetuses as well. Steps must be taken by healthcare teams to protect the mother from infection and should 
she become infected further precautions to protect the healthcare team and neonate are necessary. Personal protective equipment (PPE) for both the mother and team, particularly during delivery, are vital. Women with active infection requiring supplemental oxygen should wear a surgical mask over the nasal canula and care should be taken to prevent cross-contamination via the gas delivery system [27]. N95 use can also be used to lower risk of infection, particularly in pregnant healthcare workers; however, teams should consider the reduced tidal volume and minute ventilation secondary to N95 use, particularly during the second and third trimester [27], and alternative protections should be considered. Finally, beyond protecting patients and healthcare teams, plans need to be established ahead of time to ensure access and transportation for these services. PPE and pregnancy precautions are of course best left to the obstetric team and the patient and are not the focus of this review. However, they do serve to illustrate the importance of preparation, having the infrastructure and equipment in place ahead of time, and ensuring accessibility to obstetric services for women, especially during a pandemic such as this.

\section{Vaccination Campaign}

It is currently difficult to assess the worldwide vaccination campaign, as this has only just begun in December 2020 with the first wave of vaccines stretching into January 2021. The Pfizer and Moderna vaccines are two of the more commonly administered in western countries such as the United States. However, even now, it is apparent that there will be a delay in access to this life saving, and potentially pandemic ending, therapy in LMICs. In fact, it is predicted that at least $90 \%$ of people in 67 low-income countries have little chance of vaccination in 2021, the source citing vaccine hoarding by wealthier nations [29]. Storage and shelf life make it difficult for healthcare systems to transport, store, and administer these vaccines as well, putting an additional burden and obstacle to be overcome by less robust healthcare systems. For example, the Pfizer vaccine requires storage between -80 to $-60^{\circ} \mathrm{C}$, while the Moderna vaccine can be stored a little warmer at -25 to $-15^{\circ} \mathrm{C}$ [30]. Even when there is availability and storage arrangements have been made, cost can play a large role in accessibility. For example, in South Africa, Pfizer offered a discounted rate per dose to make purchasing the vaccine more affordable. Unfortunately, even at a discounted rate, the cost was still prohibitive [31]. There is also the concern for new viral variants emerging, such as the UK variant and South African variant. The South African variant is notable for its large number of mutations and has been noted to be more contagious than prior strains [32]. While concerning, it is important to note per a Moderna press release, that while they are noting a 6-fold reduction in neutralizing titers, levels remain above those thought to be protective [33]. While promising, South Africa itself has received supplies of the Johnson \& Johnson, Pfizer, and AstraZeneca vaccines as of February 2021. While initially thought to cover this new variant, campaign roll out has been put on hold due to disappointing results covering the South African variant [34]
While there is much working against access to vaccinations in LMICs, there is some good news as well. With regards to cost, AstraZeneca has promised to deliver vaccines, not for profit, throughout the pandemic. Their vaccine is also stated to be stable between $2-8^{\circ} \mathrm{C}$, making it more easily stored and distributed [31]. There is also concern for hesitancy with regards to vaccines. For example, in Western Europe, a 2018 survey showed only 59\% of participants believing vaccines to be safe [35]. This is in stark contrast to LMICs, where 95\% of participants in South Asia and $92 \%$ of participants in East Africa believe vaccines to be safe [35]. Fortunately, the WHO, The Coalition for Epidemic Preparedness Innovations (CEPI), and The Global Alliance for Vaccines and Immunizations (GAVI) have seen the need for vaccination worldwide and have taken steps to ensure access to the COVID-19 vaccine to the global poor, developing a program called COVAX. In the setting of this pandemic, COVAX aims for equitable access to all countries of the world, with plans to offer doses to at least $20 \%$ of the population, diverse and actively manage vaccine portfolios, ensure timely vaccine delivery, strive for the end of the acute phase of the pandemic, and to help rebuild economies [36]. While some of this is promising news for LMICs, it is important to remember that they continue to face struggles in availability and will likely not start seeing significant relief until 2022. Of note, even though most individuals in East Africa and South Asia believe vaccines to be safe, with access to programs like COVAX, they remain at significant risk when their leaders believe otherwise. In Tanzania, President Magufuli and other government officials have spoken out about the safety of these vaccines, going so far as to claim Tanzania to be a "COVID-19-free country" [37]. Going beyond simply questioning vaccine safety, Tanzania, Burundi, Eritrea, and Madagascar, are African nations that have all chosen to opt out of COVAX support at this time.

\section{Conclusion}

This is just a small example of considerations that need to be made by healthcare systems in the setting of a pandemic. Unfortunately, COVID-19 is not the first and will not be the last pandemic we have faced. The hope is that with each new disease/ outbreak we learn more and our response and capabilities continue to improve as we strive to ensure access to care for all. Services such as preventative vaccinations and disease-specific treatments must be maintained without lapses in care. In sub-Saharan Africa programs and services combating HIV/AIDS, malaria, and TB must be supported and not overlooked by local and international healthcare services. We must also ensure access to women's health services and obstetric care. Beyond simply ensuring continuity of care for these patients, we must also examine the socioeconomic effect our pandemic response places on a country. Continuing healthcare services does not help anyone if public transportation shuts down or is severely limited. Quarantine and lockdown may ensure social distancing but at what cost to a mother and child in an abusive household? In countries like the United States with a robust healthcare system these issues are less of a concern, but 
in many LMICs with limited resources, they are often forced to choose where to allocate resources between pandemic response and continued access to necessary services. In addition to resource allocation to combat the pandemic directly, considerations need to be made to ensure equitable access to vaccination for all people of the world with special attention paid to LMICs and the global poor In fact, concerning the global poor, poverty levels have now begun to rise for the first time since 1998 [38]. Estimates of where we will end up vary, with initial estimates placing the global poverty level back around 2017 levels. However, more recent numbers are far worse, stating a reversal of greater than 10 years improvements on global poverty.

The key point of this review is that no one model will work for every nation or patient population. Local healthcare services, with the help of international NGOs, need to develop their own response not just to COVID-19 but for future pandemics regarding services mentioned in this review and beyond. There is much that still needs to be examined with regards to COVID- 19, as we are still amid the pandemic and vaccination campaigns have only just begun. Research looking retroactively at the morbidity and mortality due to HIV/AIDS and other diseases in the setting of COVID-19 will be important to determine the true effect of this lockdown with the decreased access to services. Research examining the effect that this lockdown has on women's health is also important. This includes examining the change in number of unintended pregnancies, reported and unreported cases of domestic violence against women and children, and access to necessary obstetric and gynecologic care. It will also be important to retroactively examine any delays in access to vaccines and the emergence of herd immunity in LMICs. Much of this review is based on projections, therefore comparing these projections to reality after the fact will be imperative. It will be necessary for healthcare systems to establish plans, with steps in place ahead of time, so that by the time of the next pandemic we are prepared to respond immediately. This includes but is not limited to having proper facilities in place or accessible, having access to necessary equipment, and assurances for continued access to care for all.

\section{Acknowledgement}

No.

\section{Conflict of Interest}

No conflict of interest.

\section{References}

1. (2020) The devastating consequences of coronavirus lockdowns in poor countries.

2. WHO Coronavirus Disease (COVID-19) Dashboard. (2020). WHO?

3. CDC COVID Data Tracker (2020) CDC.

4. Divala T (2020) Africa faces difficult choices in responding to COVID-19. The Lancet 395: 1611

5. Country comparison- median age (2021) CIA gov.
6. Broadbent A (2020) Lockdown is not egalitarian: the costs fall on the global poor.The Lancet 396: 21-22

7. Hodgins S, Saad A (2020) Will the higher-income country blueprint for COVID-19 work in low- and lower middle-income countries? GlobHealth Sci Pract 8(2): 136-143.

8. (2020) Coronavirus: India's pandemic lockdown turns into a human tragedy. BBC News.

9. Newman T (2020) Comparing COVID-19 with previous pandemics. Medical News Today.

10. Baldo V, Bertoncello C, Cocchio S (2016) The new pandemic influenza A/ (H1N1) pdm09 virus: is it really "new"? J Prev Med Hyg 57(1): E19-E22.

11. Kucarski AJ (2020) Early dynamics of transmission and control of COVID-19: a mathematical modelling study. The Lancet Infectious Diseases 20(5): P553-558.

12. Wilder Smith A, Freedman DO (2020): Isolation, quarantine, social distancing and community containment: pivotal role for old-style public health measures in the novel coronavirus (2019-nCoV) outbreak, Journal of Travel Medicine. 27(2).

13. Pai M (2020) AIDS, TB and Malaria set to get deadlier due to coronavirus. Forbes.

14. (2020) The potential impact of health service disruptions on the burden of malaria: a modelling analysis for countries in sub-Saharan Africa. Geneva: World Health Organization.

15. Roberts L (2020) Measles is on the rise-and COVID-19 could make it worse. Nature 580: 447-448.

16. Abbas K, Procter SR (2020) Routine childhood immunisation during the COVID- 19 pandemic in Africa: a benefit-risk analysis of health benefits versus excess risk of SARS- CoV-2 infection. The Lancet 8(10): E1264-E1272.

17. (2020) We did a rapid assessment: the TB response is heavily impacted by the COVID-19 pandemic. Stop TB Partnership.

18. (2020) The potential impact of the COVID-19 response on tuberculosis in high-burden countries: a modeling analysis. Stop TB Partnership.

19. (2020) Tailoring malaria interventions in the COVID-19 response. Geneva: World Health Organization.

20. L Jewell, Britta, Mudimu, Edinah, Stover John, Kelly Sherrie L, et al (2020) Potential effects of disruption to HIV programmes in sub-Saharan Africa caused by COVID- 19: results from multiple mathematical models. figshare.

21. Roberton T (2020) Early estimates of the indirect effects of the COVID-19 pandemic on maternal and child mortality in low-income and middleincome countries: a modelling study. The Lancet, 8(7): E901-E908.

22. (2020) UN Secretary-General's policy brief: The impact of COVID-19 on women | Digital library: Publications [Internet]. UN Women.

23. Joyce R, Xu X (2020) Sector shutdowns during the coronavirus crisis: which workers are most exposed? Institute for Fiscal Studies, briefing note.

24. (2020) Pandemics and Violence Against Women and Children [Internet] Center for Global Development.

25. (2020) COVID-19 could lead to millions of unintended pregnancies, new UN-backed data reveals [Internet]. UN News.

26. Small E, Sharma BB, Nikolova SP (2020). Covid-19 and Gender in LMICs: Potential Lessons from HIV Pandemic. AIDS and behavior 24: 29952998.

27. Dashraath P, Wong JLJ, Lim MXK (2020) Coronavirus disease 2019 (COVID-19) pandemic and pregnancy. Am J Obstet Gynecol 222(6): 521531.

28. Alzamora MC, Paredes T, Caceres D, Webb CM, Valdez LM, et al. (2020) Severe COVID- 19 during Pregnancy and Possible Vertical Transmission. 
Am J Perinatol 37(8): 861- 865.

29. Dyer 0 (2020) Covid-19: Many poor countries will see almost no vaccine next year, aid groups warn. BMJ 371: m4809.

30. US COVID-19 Vaccine Product Information. CDC.

31. Boseley S (2021) Global immunisation: low-income countries rush to access Covid vaccine supply. The Guardian.

32. (2021) South Africa covid-19 strain: what we know about the new variant. The Wall Street Journal.

33. (2021) Moderna COVID-19 Vaccine Retains Neutralizing Activity Against Emerging Variants First Identified in the U.K. and the Republic of South Africa. Moderna.
34. (2021) Coronavirus: South Africa rolls out vaccine programme, BBC News.

35. Bhopal S., Nielsen M (2021) Vaccine hesitancy in low- and middleincome countries: potential implications for the COVID-19 response. Archives of Disease in Childhood 106(2): 113-114.

36.(2021) COVAX: Working for global equitable access to COVID-19 vaccines. WHO?

37. Makoni M (2021) Tanzania refuses COVID-19 vaccines. The Lancet, Pp. 397.

38. (2020) Covid-19 is undoing years of progress in curbing global poverty. The Economist. 